\title{
ON CUSTOMER FLOWS IN JACKSON QUEUEING NETWORKS
}

\author{
SEN TAN * AND \\ AIHUA XIA, ${ }^{* *}$ University of Melbourne
}

\begin{abstract}
Melamed's theorem states that, for a Jackson queueing network, the equilibrium flow along a link follows a Poisson distribution if and only if no customers can travel along the link more than once. Barbour and Brown (1996) considered the Poisson approximate version of Melamed's theorem by allowing the customers a small probability $p$ of travelling along the link more than once. In this note, we prove that the customer flow process is a Poisson cluster process and then establish a general approximate version of Melamed's theorem that accommodates all possible cases of $0 \leq p<1$.

Keywords: Jackson queueing network; Palm distribution; Poisson cluster process; Stein's method; negative binomial distribution
\end{abstract}

2010 Mathematics Subject Classification: Primary 60G55

Secondary 60F05; 60E15

\section{Introduction and the main results}

We consider a Jackson queueing network with $J$ queues and the following specifications (see Barbour and Brown (1996) for more details). First, we assume that customers can move from one queue to another, and that they can enter and leave from any queue. We assume that the exogenous arrival processes are independent Poisson processes with rates $v_{j}, 1 \leq j \leq J$. Service requirements are assumed to be exponential random variables with parameter 1 , and when there are $m$ customers in queue $j$, the service effort for queue $j$ is $\phi_{j}(m)$, where $\phi_{j}(0)=0$, $\phi_{j}(1)>0$, and $\phi_{j}(m)$ is a nondecreasing function of $m$. Second, we define the switching process as follows. Let $\lambda_{i j}$ be the probability that an individual moves from queue $i$ to queue $j$, and let $\mu_{i}$ be the exit probability from queue $i$. It is natural to assume that

$$
\sum_{j=1}^{J} \lambda_{i j}+\mu_{i}=1, \quad 1 \leq i \leq J .
$$

Without loss of generality, we may assume that the network is irreducible in the sense that all customers can access any queue with a positive probability. Set $\alpha_{j}$ as the total rate of arriving customers (including both exogenous and endogenous arrivals) to queue $j$. Then the rates $\left\{\alpha_{j}\right\}$ satisfy the equations

$$
\alpha_{j}=v_{j}+\sum_{i=1}^{J} \alpha_{i} \lambda_{i j}, \quad 1 \leq j \leq J,
$$

and they are the unique solution of the equations with $\alpha_{j}>0$ for all $j$.

Received 20 January 2010; revision received 9 July 2010.

* Postal address: Department of Mathematics and Statistics, University of Melbourne, Parkville, VIC 3052, Australia.

**Email address: xia@ms.unimelb.edu.au 
For convenience, we define state 0 as the outside of the network, that is, the point of arrival and departure of an individual into and from the system. We define $s:=\{(j, k): 0 \leq j, k \leq J\}$ as the set of all possible direct links, and we use $\tilde{\Xi}^{j k}$ to record the transitions of individuals moving from queue $j$ to queue $k$. Then $\tilde{\Xi}=\left\{\tilde{\Xi}^{j k}, 0 \leq j, k \leq J\right\}$ gives a full account of customer flows in the network, where departures are transitions to 0 and arrivals are transitions from 0 . If $\rho_{j k}$ is the rate of equilibrium flow along the link $(j, k)$ then $\rho_{j k}=\alpha_{j} \lambda_{j k}$ and the mean measure of $\tilde{\Xi}$ is

$$
\tilde{\lambda}(\mathrm{d} s,(j, k))=\rho_{j k} \mathrm{~d} s, \quad s \in \mathbb{R},(j, k) \in \& .
$$

For the point process $\tilde{\Xi}$ with locally finite mean measure $\tilde{\lambda}(\mathrm{d} s,(j, k))$, we may consider it as a random measure on the metric space $\mathbb{R} \times \&$ equipped with the metric

$$
d\left(\left(u_{1},\left(j_{1}, j_{2}\right)\right),\left(u_{2},\left(k_{1}, k_{2}\right)\right)\right)=\left|u_{1}-u_{2}\right| \mathbf{1}_{\left(j_{1}, j_{2}\right) \neq\left(k_{1}, k_{2}\right)}
$$

for $u_{1}, u_{2} \in \mathbb{R}$ and $\left(j_{1}, j_{2}\right),\left(k_{1}, k_{2}\right) \in \delta$, so that we can define the Palm distribution at $\alpha \in$ $\mathbb{R} \times \delta$ as the distribution of $\tilde{\Xi}$ conditional on the presence of a point at $\alpha$, that is,

$$
P^{\alpha}(\cdot)=\frac{\mathrm{E}\left[\mathbf{1}_{[\tilde{\Xi} \in \cdot]} \tilde{\Xi}(\mathrm{d} \alpha)\right]}{\tilde{\lambda}(\mathrm{d} \alpha)}, \quad \alpha \in \mathbb{R} \times \&, \tilde{\lambda} \text {-almost surely }
$$

see Kallenberg (1983, p. 83) for more details. A process $\tilde{\Xi}^{\alpha}$ is called the Palm process of $\tilde{\Xi}$ at $\alpha$ if its distribution is $P^{\alpha}$. In applications, it is often more convenient to work with the reduced Palm process, $\tilde{\Xi}^{\alpha}-\delta_{\alpha}$ (see Kallenberg (1983, p. 84)), where $\delta_{\alpha}$ is the Dirac measure at $\alpha$.

For two random elements $\eta_{1}$ and $\eta_{2}$ having the same distribution, we write for brevity $\eta_{1} \stackrel{\mathrm{D}}{=} \eta_{2}$.

Proposition 1.1. For each $(j, k) \in \&$, there is a point process $\tilde{\xi}^{(0,(j, k))}$ on $\mathbb{R} \times \&$ independent of $\tilde{\Xi}$ such that

$$
\tilde{\xi}^{(0,(j, k))}+\tilde{\Xi} \stackrel{\mathrm{D}}{=} \tilde{\Xi}^{(0,(j, k))} .
$$

Let $\theta_{s}, s \in \mathbb{R}$, denote the shift operator on $\mathbb{R} \times \&$ which translates each point in $\mathbb{R} \times \&$ by $s$ to the left, i.e. $\theta_{s}((u,(j, k)))=(u-s,(j, k))$, and use $\tilde{\xi}^{(s,(j, k))}$ to stand for a copy of $\tilde{\xi}^{(0,(j, k))} \circ \theta_{s}, s \in \mathbb{R}$.

Our interest is in the customer flows along the links in $C \subset \&$ for the time interval $[0, t]$, so we set the carrier space as $\Gamma_{C, t}=[0, t] \times C$. With the metric $d, \Gamma_{C, t}$ is a Polish space and $\mathcal{B}\left(\Gamma_{C, t}\right)$ denotes the Borel $\sigma$-algebra in $\Gamma_{C, t}$. Let $H_{C, t}$ denote the class of all finite nonnegative integer-valued measures on $\Gamma_{C, t}$, and let $\mathscr{H}_{C, t}$ be the $\sigma$-algebra in $H_{C, t}$ generated by the sets $\left\{\xi \in H_{C, t}: \xi(B)=i\right\}, i \in \mathbb{Z}_{+}:=\{0,1,2, \ldots\}$, and $B \in \mathcal{B}\left(\Gamma_{C, t}\right)$ (see Kallenberg (1983, p. 12)).

We use $\tilde{\Xi}_{C, t}$ to denote the transitions along the links in $C$ for the period $[0, t]$. Then the mean measure of $\tilde{\Xi}_{C, t}$ is

$$
\tilde{\lambda}_{C, t}(\mathrm{~d} s,(j, k))=\rho_{j k} \mathrm{~d} s, \quad 0 \leq s \leq t,(j, k) \in C .
$$

Melamed's theorem states that $\tilde{\Xi}_{C, t}$ is a Poisson process if and only if no customers travel along the links in $C$ more than once. Barbour and Brown (1996) considered the Poisson approximate version of Melamed's theorem by allowing the customers a small probability (loop probability) of travelling along the links more than once, and they concluded that the accuracy of the Poisson approximation depends on how small the loop probability is. 
Theorem 1.1. The process $\tilde{\Xi}_{C, t}$ is a Poisson cluster process. More precisely, let $\left\{\eta_{i}, i \geq 0\right\}$ be independent and identically distributed random measures on $\Gamma_{C, t}$ having the distribution

$$
\begin{aligned}
\mathrm{P}\left[\eta_{0}\left(\Gamma_{C, t}\right) \geq 1\right] & =1, \\
\mathrm{P}\left(\eta_{0} \in A\right) & =\mathrm{E} \sum_{(j, k) \in C} \int_{0}^{t} \frac{\left.\mathbf{1}_{\left[\tilde{\xi}^{(s,(j, k))} \mid\right.} \Gamma_{C, t} \in A\right]}{\tilde{\xi}^{(s,(j, k))}\left(\Gamma_{C, t}\right)} \frac{\rho_{j k}}{\theta_{C, t}} \mathrm{~d} s, \quad A \in \mathcal{H}_{C, t},
\end{aligned}
$$

where $\left.\tilde{\xi}^{(s,(j, k))}\right|_{\Gamma_{C, t}}$ is the restriction of $\tilde{\xi}^{(s,(j, k))}$ to $\Gamma_{C, t}$ (see Kallenberg $\left.(1983, p .12)\right)$ and

$$
\theta_{C, t}=\mathrm{E} \sum_{(j, k) \in C} \int_{0}^{t} \frac{1}{\tilde{\xi}^{(s,(j, k))}\left(\Gamma_{C, t}\right)} \rho_{j k} \mathrm{~d} s .
$$

Let $M$ be a Poisson random variable with mean $\theta_{C, t}$ and independent of $\left\{\eta_{i}, i \geq 0\right\}$. Then

$$
\tilde{\Xi}_{C, t} \stackrel{\mathrm{D}}{=} \sum_{i=1}^{M} \eta_{i}
$$

Despite the fact that $\theta_{C, t}$ is specified by (1.2), since the Palm process $\tilde{\xi}^{(s,(j, k))}$ is generally intractable, it is virtually impossible to express $\theta_{C, t}$ explicitly in terms of the specifications of the Jackson queueing network. We now consider a general approximate version of Melamed's theorem. Noting that $(\mathbb{R} \times \delta, d)$ is a Polish space and that, for each bounded Borel subset $B$ of $\mathbb{R} \times \&$,

$$
\mathrm{E}[\tilde{\Xi}(B)]^{2}=\mathrm{E} \int_{B} \tilde{\Xi}(B) \tilde{\Xi}(\mathrm{d} \alpha)=\mathrm{E} \int_{B} \tilde{\Xi}^{\alpha}(B) \tilde{\lambda}(\mathrm{d} \alpha) \geq \mathrm{E} \int_{B}(\tilde{\Xi}(B)+1) \tilde{\lambda}(\mathrm{d} \alpha),
$$

we obtain $\operatorname{var}[\tilde{\Xi}(B)] \geq E \tilde{\Xi}(B)$. Hence, suitable approximate models for the distribution of $\Xi_{C, t}:=\tilde{\Xi}_{C, t}\left(\Gamma_{C, t}\right)$ should necessarily possess the same property. We consider the negative binomial, $\mathrm{NB}(r, q), r>0$ and $0<q<1$, with distribution function

$$
\pi_{i}=\frac{\Gamma(r+i)}{\Gamma(r) i !} q^{r}(1-q)^{i}, \quad i \in \mathbb{Z}_{+},
$$

as the approximate distribution. The advantage of using the negative binomial approximation is that it suffices to estimate the mean and variance for the approximate distribution, as is often done when applying the central limit theorem based on the normal approximation.

To state the error estimate of the approximate version of Melamed's theorem, we define

$$
\epsilon_{C}(j, k)=\mathrm{E} \tilde{\xi}^{(0,(j, k))}(\mathbb{R} \times C)-1 \quad \text { and } \quad \epsilon_{C}=\sum_{(j, k) \in C} \frac{\rho_{j k}}{\rho_{C}} \epsilon_{C}(j, k) .
$$

In other words, $\epsilon_{C}(j, k)$ is the average number of visits in $C$ by the extra customer crossing the link $(j, k)$ and $\epsilon_{C}$ is the weighted average number of visits by an extra customer crossing links in $C$. We also need

$$
\sigma_{C}(j, k)=\mathrm{E}\left[\tilde{\xi}^{(0,(j, k))}(\mathbb{R} \times C)\left(\tilde{\xi}^{(0,(j, k))}(\mathbb{R} \times C)-1\right)\right] \quad \text { and } \quad \sigma_{C}=\sum_{(j, k) \in C} \frac{\rho_{j k}}{\rho_{C}} \sigma_{C}(j, k) .
$$

That is, $\sigma_{C}(j, k)$ is the second factorial moment of the number of visits in $C$ by the extra customer crossing the link $(j, k)$ and $\sigma_{C}$ is the weighted average of the second factorial moments of the 
number of visits by an extra customer crossing links in $C$. Let $w_{C}(j, k)$ be the probability that a link $(j, k)$ crossing customer has never crossed links in $C$ before and will not pay more visits in $C$ in the future. Define

$$
w_{C}=\sum_{(j, k) \in C} \frac{w_{C}(j, k) \rho_{j k}}{\rho_{C}},
$$

the weighted probability of customers crossing links in $C$ only once.

Theorem 1.2. Let

$$
r=\frac{\left(\rho_{C} t\right)^{2}}{\operatorname{var}\left(\Xi_{C, t}\right)-\rho_{C} t}, \quad q=\frac{\rho_{C} t}{\operatorname{var}\left(\Xi_{C, t}\right)} .
$$

Then

$$
\begin{aligned}
d_{\mathrm{TV}}\left(\Xi_{C, t}, \mathrm{NB}(r, q)\right) & :=\sup _{A \subset \mathbb{Z}_{+}}\left|\mathrm{P}\left(\Xi_{C, t} \in A\right)-\mathrm{NB}(r, q)(A)\right| \\
& \leq \frac{1}{\sqrt{2 \mathrm{e} w_{C} \rho_{C} t}}\left(2 \epsilon_{C}^{2}+\sigma_{C}\right) .
\end{aligned}
$$

Remark 1.1. If the loop probability in $C$ is 0 then the negative binomial is reduced to a Poisson distribution and the upper bound in Theorem 1.2 becomes 0 . This implies half of Melamed's (1979) theorem.

Remark 1.2. If the loop probability is between 0 and 1 , then both $\epsilon_{C}$ and $\sigma_{C}$ are finite, so the negative binomial approximation error bound is of order $O(1 / \sqrt{t})$. Furthermore, if the loop probability is small then both $\epsilon_{C}$ and $\sigma_{C}$ are small, so the negative binomial approximation to the distribution of $\Xi_{C, t}$ is even faster.

Example 1.1. To see how the loop probability appears in the upper bound, let us consider the tandem M/M/1/ $\infty$ queues with feedback investigated in Barbour and Brown (1996). Let $J=2, \phi_{1}(n)=\phi_{2}(n)=1$ for all $n \geq 1, \lambda_{12}=1, \lambda_{22}=1-\mu_{2}=p, v_{1}<1-p$, $v_{2}=0$, and $C=\{(1,2),(2,2)\}$. Direct computation gives $\rho_{12}=v_{1}, \rho_{22}=v_{1} p /(1-p)$, $\epsilon_{C}(1,2)=p /(1-p), \epsilon_{C}(2,2)=\left(1+p^{2}\right) /(1-p), \sigma_{C}(1,2)=2 p /(1-p)^{2}$, and $\sigma_{C}(2,2)=$ $2\left(1+p+p^{3}\right) /(1-p)^{2}$, so $\epsilon_{C} \leq 2 p /(1-p)$ and $\sigma_{C}=\left(4 p+2 p^{4}\right) /(1-p)^{2}$. Moreover, $w_{C}(1,2)=1-p, w_{C}(2,2)=0$, and $w_{C}=(1-p)^{2}$. Therefore, the upper bound of $(1.3)$ becomes $\left(4 p+8 p^{2}+2 p^{4}\right)(1-p)^{-2.5}\left(2 \mathrm{e} v_{1} t\right)^{-1 / 2}$.

\section{The proofs}

Proof of Proposition 1.1. The proof is adapted from Barbour and Brown (1996, p. 480). By Lemma 1 of Barbour and Brown (1996), the reduced Palm process $\tilde{\Xi}^{(0,(j, k))}-\delta_{(0,(j, k))}$ has the same distribution as that of $\tilde{\Xi}$, except that the network on $(0, \infty)$ behaves as if there were an extra individual at queue $k$ at time 0 and the network on $(-\infty, 0)$ behaves as if there were an extra individual in queue $j$ at time 0 . Whenever the extra customer is at queue $i$ with the other $m$ customers, we use independently sampled exponential service requirements with instantaneous service rate $\phi_{i}(m+1)-\phi_{i}(m)$. Noting that this construction ensures that the extra customer uses the 'spare' service effort and never 'interferes' with the flow of the main traffic, we can see that its transitions are independent of $\tilde{\Xi}$. The same procedure applies to the construction of the backward route. Let $\tilde{\xi}^{(0,(j, k))}$ be the transitions taken by the extra customer on $(-\infty, 0) \cup(0, \infty)$ plus the Dirac measure $\delta_{(0,(j, k))}$. Then $\tilde{\xi}^{(0,(j, k))}$ is independent of $\tilde{\Xi}$ and the conclusion of the lemma follows from the construction. 
Proof of Theorem 1.1. By Theorem 11.2 of Kallenberg (1983) and Proposition 1.1, we can conclude that $\tilde{\Xi}_{C, t}$ is infinitely divisible; hence, we obtain from Lemma 6.6 and Theorem 6.1 of Kallenberg (1983) that $\tilde{\Xi}_{C, t}$ is a Poisson cluster process, that is,

$$
\tilde{\Xi}_{C, t} \stackrel{\mathrm{D}}{=} \sum_{i=1}^{M} \eta_{i}
$$

where the $\eta_{i}, i \geq 0$, are independent and identically distributed random measures on $\Gamma_{C, t}$ such that $\mathrm{P}\left[\eta_{0}\left(\Gamma_{C, t}\right) \geq 1\right]=1$, and $M$ is a Poisson random variable with mean $\theta_{C, t}$, independent of $\left\{\eta_{i}, i \geq 1\right\}$. The direct verification ensures that the Palm process of $\sum_{i=1}^{M} \eta_{i}$ at $\alpha \in \Gamma_{C, t}$ is $\sum_{i=1}^{M} \eta_{i}+\eta_{0}^{\alpha}$, where $\eta_{0}^{\alpha}$ is the Palm process of $\eta_{0}$ at $\alpha$, independent of $\left\{M, \eta_{i}, i \geq 1\right\}$. This in turn implies that $\left.\tilde{\xi}^{(s,(j, k))}\right|_{\Gamma_{C, t}} \stackrel{\mathrm{D}}{=} \eta_{0}^{(s,(j, k))}$.

Let $\tilde{\mu}(\mathrm{d} s,(j, k))$ denote the mean measure of the point process $\eta_{0}$. Then some elementary computation ensures that the mean measure of $\sum_{i=1}^{M} \eta_{i}$ is $\theta_{C, t} \tilde{\mu}(\mathrm{d} s,(j, k))$ for $(j, k) \in C$ and $0 \leq s \leq t$. On the other hand, the mean measure of $\tilde{\Xi}_{C, t}$ is $\tilde{\lambda}_{C, t}(\mathrm{~d} s,(j, k))=\rho_{j k} \mathrm{~d} s$ for $(j, k) \in C$ and $s \in[0, t]$, so we obtain

$$
\tilde{\mu}(\mathrm{d} s,(j, k))=\frac{\rho_{j k}}{\theta_{C, t}} \mathrm{~d} s, \quad(j, k) \in C, s \in[0, t] .
$$

Representation (1.1) follows from the fact that $\mathrm{P}\left[\eta_{0}\left(\Gamma_{C, t}\right) \geq 1\right]=1$ and

$$
\mathrm{P}\left[\eta_{0} \in A\right]=\mathrm{E} \int_{\Gamma_{C, t}} \frac{\mathbf{1}_{\left[\eta_{0} \in A\right]}}{\eta_{0}\left(\Gamma_{C, t}\right)} \eta_{0}(\mathrm{~d} \alpha)=\mathrm{E} \sum_{(j, k) \in C} \int_{0}^{t} \frac{\left.\mathbf{1}_{\left[\tilde{\xi}^{(s,(j, k))}\right.}\right|_{\left.\Gamma_{C, t} \in A\right]}}{\tilde{\xi}^{(s,(j, k))}\left(\Gamma_{C, t}\right)} \frac{\rho_{j k}}{\theta_{C, t}} \mathrm{~d} s .
$$

In particular, if we take $A=H_{C, t}$ then the left-hand side becomes 1 , so (1.2) follows.

The proof of Theorem 1.2 is based on the following lemma.

Lemma 2.1. We have $d_{\mathrm{TV}}\left(\Xi_{C, t}, \Xi_{C, t}+1\right) \leq 1 / \sqrt{2 \mathrm{e} w_{C} \rho_{C} t}$.

Proof. We prove the claim by a coupling based on the 'priority principle' (cf. the proof of Proposition 1.1). We refer to a customer as a single crossing (SC) customer if the customer crosses links in $C$ only once, otherwise, the customer is referred to as a multiple crossing (MC) customer. We 'manage' the network by regrouping the customers at each queue into SC customers and MC customers. Whenever there are $m_{2} \mathrm{MC}$ customers together with $m_{1}$ SC customers at queue $j$, we use independently sampled exponential service requirements with instantaneous service rate $\phi_{j}\left(m_{1}+m_{2}\right)-\phi_{j}\left(m_{1}\right)$ for all of the MC customers, while the service for the SC customers is carried out with instantaneous service rate $\phi_{j}\left(m_{1}\right)$, that is, as if there are no MC customers present in the queue. Since the SC customers take priority over the MC customers and the MC customers use the 'spare' service effort and never interrupt the traffic flow of the SC customers, we can see that the transitions made by the MC customers are independent of the transitions of the SC customers. Let $Z_{1}^{j k}$ and $Z_{2}^{j k}$ respectively denote the transitions of SC and MC customers moving from queue $j$ to queue $k$ in the period $[0, t]$. Then $\tilde{Z}_{1}:=\left\{Z_{1}^{j k},(j, k) \in C\right\}$ and $\tilde{Z}_{2}:=\left\{Z_{2}^{j k},(j, k) \in C\right\}$ are independent, and

$$
\tilde{\Xi}_{C, t} \stackrel{\mathrm{D}}{=} \tilde{Z}_{1}+\tilde{Z}_{2} \text {. }
$$

By Melamed's theorem, the point process $\tilde{Z}_{1}$ is a Poisson process with mean measure

$$
\tilde{\lambda}_{\tilde{Z}_{1}}(\mathrm{~d} s,(j, k))=w_{C}(j, k) \rho_{j k} \mathrm{~d} s, \quad(j, k) \in C, 0 \leq s \leq t,
$$


so $\tilde{Z}_{1}\left(\Gamma_{C, t}\right)$ follows a Poisson distribution with mean $w_{C} \rho_{C} t$ and Proposition A.2.7 of Barbour et al. (1992, p. 262) ensures that

$$
d_{\mathrm{TV}}\left(\Xi_{C, t}, \Xi_{C, t}+1\right) \leq d_{\mathrm{TV}}\left(\tilde{Z}_{1}\left(\Gamma_{C, t}\right), \tilde{Z}_{1}\left(\Gamma_{C, t}\right)+1\right) \leq \frac{1}{\sqrt{2 \mathrm{e} w_{C} \rho_{C} t}} .
$$

This completes the proof.

Proof of Theorem 1.2. Let $a=r(1-q)$ and $b=1-q$. Brown and Xia (2001) defined a generator for $\mathrm{NB}(r, q)$ as

$$
\mathcal{B} g(i)=(a+b i) g(i+1)-i g(i) \quad \text { for } i \in \mathbb{Z}_{+} \text {and a function } g \text { on } \mathbb{Z}_{+},
$$

and established the Stein identity

$$
\mathscr{B} g(i)=f(i)-\pi(f)
$$

for $f \in \mathcal{F}:=\left\{f: \mathbb{Z}_{+} \rightarrow[0,1]\right\}$, where $\pi(f)=\sum_{i=0}^{\infty} f(i) \pi_{i}$. It was shown in Brown and Xia (2001) that, for each $f \in \mathcal{F}$, the solution $g_{f}$ to the Stein equation (2.1) satisfies

$$
\left\|\Delta g_{f}\right\| \leq \frac{1}{a}
$$

where $\Delta g_{f}(\cdot)=g_{f}(\cdot+1)-g_{f}(\cdot)$. The Stein identity (2.1) ensures that

$$
\sup _{f \in \mathcal{F}}\left|\mathrm{E} f\left(\Xi_{C, t}\right)-\pi(f)\right|=\sup _{f \in \mathcal{F}}\left|\mathrm{E} \mathscr{B} g_{f}\left(\Xi_{C, t}\right)\right| ;
$$

hence, it suffices to estimate $\mathrm{E} \mathscr{B} g_{f}\left(\Xi_{C, t}\right)$ for all $f \in \mathcal{F}$. For convenience, we drop the subscript $f$ from $g_{f}$. By Proposition 1.1 we can take a point process $\tilde{\xi}_{C, t}^{(s,(j, k))}$ on $\Gamma_{C, t}$ independent of $\tilde{\Xi}_{C, t}$ such that

$$
\tilde{\Xi}_{C, t}^{(s,(j, k))}=\tilde{\Xi}_{C, t}+\tilde{\xi}_{C, t}^{(s,(j, k))} .
$$

Therefore, if we write $\tilde{\xi}_{C, t}^{(s,(j, k))}\left(\Gamma_{C, t}\right)=1+\xi^{(s,(j, k))}$ then

$$
\begin{aligned}
\mathrm{E} \mathscr{B} g\left(\Xi_{C, t}\right)= & a \operatorname{Eg}\left(\Xi_{C, t}+1\right)+b \sum_{(j, k) \in C} \int_{0}^{t} g\left(\Xi_{C, t}+2+\xi^{(s,(j, k))}\right) \rho_{j k} \mathrm{~d} s \\
& -\sum_{(j, k) \in C} \int_{0}^{t} g\left(\Xi_{C, t}+1+\xi^{(s,(j, k))}\right) \rho_{j k} \mathrm{~d} s .
\end{aligned}
$$

Let $a+(b-1) \rho_{C} t=0$ and $\tilde{\Xi}_{C, t}=\Xi_{C, t}+1$. Then it follows from (2.3) that

$$
\begin{gathered}
\mathrm{E} \mathscr{B} g\left(\Xi_{C, t}\right)=\mathrm{E} \sum_{(j, k) \in C} \int_{0}^{t}\left[b\left(g\left(\tilde{\Xi}_{C, t}+1+\xi^{(s,(j, k))}\right)-g\left(\tilde{\Xi}_{C, t}\right)\right)\right. \\
\left.-\left(g\left(\tilde{\Xi}_{C, t}+\xi^{(s,(j, k))}\right)-g\left(\tilde{\Xi}_{C, t}\right)\right)\right] \rho_{j k} \mathrm{~d} s \\
=\mathrm{E} \sum_{(j, k) \in C} \int_{0}^{t}\left\{\sum_{r=0}^{\xi^{(s,(j, k))}-1}\left[b \Delta g\left(\tilde{\Xi}_{C, t}+r+1\right)-\Delta g\left(\tilde{\Xi}_{C, t}+r\right)\right]\right. \\
\left.+b \Delta g\left(\tilde{\Xi}_{C, t}\right)\right\} \rho_{j k} \mathrm{~d} s .
\end{gathered}
$$


Now, set

$$
b=\frac{\sum_{(j, k) \in C} \int_{0}^{t} \mathrm{E} \xi^{(s,(j, k))} \rho_{j k} \mathrm{~d} s}{\sum_{(j, k) \in C} \int_{0}^{t} \mathrm{E} \xi^{(s,(j, k))} \rho_{j k} \mathrm{~d} s+\rho_{C} t}=\frac{\operatorname{var}\left(\Xi_{C, t}\right)-\rho_{C} t}{\operatorname{var}\left(\Xi_{C, t}\right)},
$$

where the last equality is due to

$$
\begin{aligned}
\mathrm{E} \Xi_{C, t}^{2} & =\mathrm{E} \int_{\Gamma_{C, t}} \Xi_{C, t} \tilde{\Xi}_{C, t}(\mathrm{~d} \alpha) \\
& =\sum_{(j, k) \in C} \mathrm{E} \int_{0}^{t}\left(\Xi_{C, t}+1+\xi^{(s,(j, k))}\right) \rho_{j k} \mathrm{~d} s \\
& =\left(\mathrm{E} \Xi_{C, t}\right)^{2}+\rho_{C} t+\mathrm{E} \sum_{(j, k) \in C} \int_{0}^{t} \xi^{(s,(j, k))} \rho_{j k} \mathrm{~d} s,
\end{aligned}
$$

and so

$$
\mathrm{E} \sum_{(j, k) \in C} \int_{0}^{t} \xi^{(s,(j, k))} \rho_{j k} \mathrm{~d} s=\operatorname{var}\left(\Xi_{C, t}\right)-\rho_{C} t .
$$

Using the fact that $\xi^{(s,(j, k))}$ is independent of $\tilde{\Xi}_{C, t}$, we obtain, from (2.4),

$$
\begin{aligned}
\mathrm{E} \mathscr{B} g\left(\Xi_{C, t}\right)=\mathrm{E} \sum_{(j, k) \in C} \int_{0}^{t}\left\{\sum_{r=0}^{\xi^{(s,(j, k))}-1}\right. & {\left[b \mathrm{E} \Delta^{2} g\left(\tilde{\Xi}_{C, t}+r\right)\right.} \\
& \left.\left.-(1-b) \sum_{l=0}^{r-1} \mathrm{E} \Delta^{2} g\left(\tilde{\Xi}_{C, t}+l\right)\right]\right\} \rho_{j k} \mathrm{~d} s .
\end{aligned}
$$

On the other hand, we apply (2.2) and Lemma 2.1 to obtain

$$
\left|\mathrm{E} \Delta^{2} g\left(\tilde{\Xi}_{C, t}+l\right)\right| \leq 2\|\Delta g\| d_{\mathrm{TV}}\left(\Xi_{C, t}, \Xi_{C, t}+1\right) \leq \frac{2}{a \sqrt{2 \mathrm{e} w_{C} \rho_{C} t}},
$$

which in turn implies that

$$
\begin{aligned}
\left|\mathrm{E} \mathscr{B} g\left(\Xi_{C, t}\right)\right| \leq & \left(a \sqrt{2 \mathrm{e} w_{C} \rho_{C} t}\right)^{-1} \\
& \times \sum_{(j, k) \in C} \int_{0}^{t}\left[2 b \mathrm{E} \xi^{(s,(j, k))}+(1-b) \mathrm{E} \xi^{(s,(j, k))}\left(\xi^{(s,(j, k))}-1\right)\right] \rho_{j k} \mathrm{~d} s .
\end{aligned}
$$

Finally, (1.3) follows from the definitions of $\epsilon_{C}$ and $\sigma_{C}$ since

$$
\mathrm{E} \sum_{(j, k) \in C} \int_{0}^{t} \xi^{(s,(j, k))} \rho_{j k} \mathrm{~d} s \leq \epsilon_{C} \rho_{C} t
$$

and

$$
\sum_{(j, k) \in C} \int_{0}^{t} \mathrm{E} \xi^{(s,(j, k))}\left(\xi^{(s,(j, k))}-1\right) \rho_{j k} \mathrm{~d} s \leq \sigma_{C} \rho_{C} t .
$$

This completes the proof. 


\section{References}

Barbour, A. D. and Brown, T. C. (1996). Approximate versions of Melamed's theorem. J. Appl. Prob. 33, 472-489. Barbour, A. D., Holst, L. and Janson, S. (1992). Poisson Approximation. Oxford University Press.

Brown, T. C. And Xia, A. (2001). Stein's method and birth-death processes. Ann. Prob. 29, 1373-1403.

Kallenberg, O. (1983). Random Measures, 3rd edn. Academic Press, London.

Melamed, B. (1979). Characterizations of Poisson traffic streams in Jackson queueing networks. Adv. Appl. Prob. 11, 422-438. 